\title{
DeepSNVMiner: A sequence analysis tool to detect emergent, rare mutations in subsets of cell populations
}

\author{
T. Daniel Andrews, Yogesh Jeelall, Dipti Talaulikar, Christopher C. Goodnow, Matthew A Field
}

Background. Massively parallel sequencing technology is being used to sequence highly diverse populations of DNA such as that derived from heterogeneous cell mixtures containing both wild-type and disease-related states. At the core of such molecule tagging techniques is the tagging and identification of sequence reads derived from individual input DNA molecules, which must be first computationally disambiguated to generate read groups sharing common sequence tags, with each read group representing a single input DNA molecule. This disambiguation typically generates huge numbers of reads groups, each of which requires additional variant detection analysis steps to be run specific to each read group, thus representing a significant computational challenge. While sequencing technologies for producing these data are approaching maturity, the lack of available computational tools for analysing such heterogeneous sequence data represents an obstacle to the widespread adoption of this technology.

Results. Using synthetic data we successfully detect unique variants at dilution levels of 1 in a $1,000,000$ molecules, and find DeeepSNVMiner obtains significantly lower false positive and false negative rates compared to popular variant callers GATK, SAMTools, FreeBayes and LoFreq, particularly as the variant concentration levels decrease. In a dilution series with genomic DNA from two cells lines, we find DeepSNVMiner identifies a known somatic variant when present at concentrations of only 1 in 1,000 molecules in the input material, the lowest concentration amongst all variant callers tested.

Conclusions. Here we present DeepSNVMiner; a tool to disambiguate tagged sequence groups and robustly identify sequence variants specific to subsets of starting DNA molecules that may indicate the presence of a disease. DeepSNVMiner is an automated workflow of custom sequence analysis utilities and open source tools able to differentiate somatic DNA variants from artefactual sequence variants that likely arose during DNA amplification. The workflow remains flexible such that it may be customised to variants of the data production protocol used - and supports reproducible analysis through detailed logging and reporting of results. DeepSNVMiner is available for academic non-commercial research purposes at https://github.com/mattmattmattmatt/DeepSNVMiner . 


\section{DeepSNVMiner: A sequence analysis tool to detect emergent, 2 rare mutations in subsets of cell populations}

3 T. Daniel Andrews ${ }^{1,2}$, Yogesh Jeelal ${ }^{1}$, Dipti Talaulikar ${ }^{1,3,4}$, Christopher C. Goodnow ${ }^{1,5,{ }^{*}}$ \&

4 Matthew A. Field ${ }^{1,2, *}$

$5{ }^{1}$ Immunogenomics Laboratory, Department of Immunology, John Curtin School of Medical

6 Research, Australian National University, Canberra 2600, Australia

$7 \quad{ }^{2}$ National Computational Infrastructure, Australia National University, Canberra 2600, Australia

$8{ }^{3}$ Haematology Department, Canberra Hospital, Canberra 2600, Australia

$9 \quad{ }^{4}$ ANU Medical School, Australian National University, Canberra 2600, Australia

$10{ }^{5}$ Immunogenomics Laboratory, Immunology Division, Garvan Institute of Medical Research,

11 Darlinghurst 2010, Australia

12

$13 *$ Equal senior authors

15 Corresponding author:

16 Matthew Field

17 GPO Box 334, Canberra City, ACT, 2601, Australia

18 Email address: matt.field@anu.edu.au 


\section{Abstract}

24 Background. Massively parallel sequencing technology is being used to sequence highly diverse

25 populations of DNA such as that derived from heterogeneous cell mixtures containing both wild-

26 type and disease-related states. At the core of such molecule tagging techniques is the tagging

27 and identification of sequence reads derived from individual input DNA molecules, which must

28 be first computationally disambiguated to generate read groups sharing common sequence tags,

29 with each read group representing a single input DNA molecule. This disambiguation typically

30 generates huge numbers of reads groups, each of which requires additional variant detection

31 analysis steps to be run specific to each read group, thus representing a significant computational

32 challenge. While sequencing technologies for producing these data are approaching maturity,

33 the lack of available computational tools for analysing such heterogeneous sequence data

34 represents an obstacle to the widespread adoption of this technology.

36 Results. Using synthetic data we successfully detect unique variants at dilution levels of 1 in a

37 1,000,000 molecules, and find DeepSNVMiner obtains significantly lower false positive and

38 false negative rates compared to popular variant callers GATK, SAMTools, FreeBayes and

39 LoFreq, particularly as the variant concentration levels decrease. In a dilution series with

40 genomic DNA from two cells lines, we find DeepSNVMiner identifies a known somatic variant

41 when present at concentrations of only 1 in 1,000 molecules in the input material, the lowest

42 concentration amongst all variant callers tested.

44 Conclusions. Here we present DeepSNVMiner; a tool to disambiguate tagged sequence groups and robustly identify sequence variants specific to subsets of starting DNA molecules that may 
46 indicate the presence of a disease. DeepSNVMiner is an automated workflow of custom

47 sequence analysis utilities and open source tools able to differentiate somatic DNA variants from

48 artefactual sequence variants that likely arose during DNA amplification. The workflow remains

49 flexible such that it may be customised to variants of the data production protocol used - and

50 supports reproducible analysis through detailed logging and reporting of results. DeepSNVMiner

51 is available for academic non-commercial research purposes at

52 https://github.com/mattmattmattmatt/DeepSNVMiner.

\section{Introduction}

63

Deep sequencing of a restricted set of gene targets in a large population of cells has rapidly become a key application of second-generation sequencing - allowing a census of variation to be conducted on an in vivo biological system (Fu et al. 2011; Hiatt et al. 2013; Jabara et al. 2011; Kinde et al. 2011; Kivioja et al. 2012; Schmitt et al. 2012). Applications of this technology allow polling of sequence variation in cancer subtypes (Forshew et al. 2012), ascertainment of minimal residual disease (Bidard et al. 2013), ascertainment of malignancies or antibody specificity in the immune system (Georgiou et al. 2014) and observation of the emergence of drug resistant virus point-mutants (Al-Mawsawi et al. 2014).

The central technique in molecule tagging that allows disambiguation of these deep sequence datasets is the attachment of a random unique sequence identifier (UID) to the end(s) of input DNA, either prior to or simultaneously with amplification of target sequences (Fig. 1). Hence, even though subsequent polymerase amplification of target sequences may introduce errors, mapping these sequences to their UID sequence allows easy differentiation of sequence variation 
69 that was originally present in the input DNA from variation that has been introduced during

70 subsequent amplification steps. Recently developed methods for molecule tagging rely on

71 digital PCR, a process where individual DNA molecules are assessed individually (Vogelstein \&

72 Kinzler 1999). Several variants of this technique have now been described (Dressman et al. 2003;

73 Ottesen et al. 2006) with the common thread being the binding of oligonucleotide to each

74 individual input DNA molecule prior to or during amplification. This technique is not to be

75 confused with sample barcoding or multiplexing, a process where individual samples are tagged

76 with small oligonucleotides and pooled in a single lane for sequencing.

In comparison to traditional massively parallel sequencing, molecule tagging has an additional step where a small unique oligonucleotide is attached to each DNA molecule prior to polymerase chain reaction (PCR) amplification. While both techniques generate huge numbers of sequenced

DNA molecules in parallel a potential issue with traditional sequencing is that the introduction of erroneous base calls into a single DNA molecule can result in inaccurate sequence information being amplified in subsequent PCR steps. Such issues are not necessary prohibitive for reliable variant detection when samples are relatively homogeneous however, mainly due to the relatively low base error and PCR bias rates (Ross et al. 2013; Schirmer et al. 2015), and the ability to remove candidate PCR duplicates reads using tools such as SAMTools (Li et al. 2009) or SAMBLASTER (Faust \& Hall 2014). When the samples being sequenced are heterogeneous however, traditional variant detection methods often fail to reliably detect rare variants due to the small fraction of the original material containing the variant of interest and differences in the variant detection algorithms (Field et al. 2015). With molecule tagging techniques, we are able to

91 overcome PCR issues and detect rare variants within heterogeneous samples due to the 
92 attachment of UIDs, effectively allowing the differentiation of amplification error from variation

93 present in the original DNA molecules (Kinde et al. 2011).

95 While the utility of sequencing tagged samples is clear, the analysis of sequence data generated with UID tags is non-trivial and, as yet, software or a computational workflow does not exist in

97 the public domain to allow easy calling and tallying of this mutation information. The fundamental technical challenge of working with such data is largely due to the wide variety of methods for attaching UIDs, methods that generate vastly different UIDs with regard to total

100 sequence length and their position on the molecules relative to the sequence of interest and/or

101 adaptors. The ability to work with such data requires software where users can first define the

102 nature of the specific UID in their experiment, followed by an analysis workflow where UIDs are

103 temporarily removed from raw sequence data for the alignment step and later restored as a means

104 of grouping the individual reads by common UID. Finally, variants must be called within each

105 group of input molecules sharing a common UID, a computationally intensive task given the

106 huge numbers of groups often generated in a single experiment. To address this need we present

107 DeepSNVMiner, a tool able to detect rare single nucleotide variants and small indels specific to a

108 single amplified DNA molecule identified by a unique tagged sequence identifier. The

109 DeepSNVMiner workflow consists of grouping reads by UID tag sequences and calling variant

110 bases in UID groups, thus identifying mutations that existed in single molecules from the

111 original heterogeneous input DNA. DeepSNVMiner is a standalone-automated workflow that

112 runs in a Linux or Macintosh environment and has been successfully used even on modest

113 desktop hardware. 


\section{Materials \& Methods}

\section{Cell lines used}

117 Two cell lines were utilised in the dilution series experiment, HEK293 and OCI-LY10. HEK293

118 is available from ATCC (accession CRL-1573; http://www.atcc.org/products/all/CRL-1573.aspx)

119 and OCI-LY10 from Ontario Cancer Institute (accession CVCL_8795;

120 https://www.abmgood.com/OCI-Ly10-Cell-Lysate-Data-Sheet-L134.html)

121

\section{Software Input}

123 Running DeepSNVMiner requires three input files; paired-end FASTQ read files and a BED file

124 containing the specific locations of targeted genomic region(s). An initial configuration step is

125 also required to determine the location of three required external resources; Burrows-Wheeler

126 Aligner (BWA) (Li \& Durbin 2009), SAMtools (Li et al. 2009), and a reference genome FASTA

127 file with BWA index files.

128

129 Workflow design

130 The workflow to disambiguate sequence variants from their unique sequence ID tags groups

131 involves multiple steps involving both purpose built tools and calls to external binaries (Fig. 2).

132 First, the sequence read dataset is subjected to preliminary quality control, to remove low quality

133 reads or those containing predominantly $\mathrm{N}$ calls (and hence avoid assigning UID groups of

134 consecutive N's). The data is next interrogated for the presence of obvious adaptor sequence,

135 which may contaminate UID tags if left untrimmed. Each UID tag is then identified based on the

136 user defined input and removed from the FASTQ sequence line and appended to the existing 
137 FASTQ read header. These filtered reads and headers are written to new FASTQ files with the

138 UID header information later used to detect variants specific to common UID groups.

139 DeepSNVMiner is flexible with regard to the structure of the UID tag as both the expected UID

140 length and strand location of the UID typically vary depending on the tagging protocol and/or

141 sequencing technology used. For example, frequently the UID is appended solely at the 5' end of

142 the amplified region, but in other protocols the sequence from both the $5^{\prime}$ and the $3^{\prime}$ ends needs to

143 be concatenated to derive the UID. Next, the modified reads are aligned to a reference genome

144 sequence with BWA (Li \& Durbin 2009) using a set of alignment parameters that are permissive

145 of mismatches but which penalise opening a gap within the alignment, especially at the ends of

146 sequence reads. Variant bases are then identified base-by-base using the SAMTools calmd

147 command ( $\mathrm{Li}$ et al. 2009) within a predefined set of user-specified target genomic locations input

148 from a BED-format file. SAMtools calmd is used instead of the more standard

149 SAMtools/BCFtools workflow, as running the millions of common UID groups we typically

150 observe through the entire SAMtools/BCFtools workflow is computationally prohibitive. Output

151 from calmd is next parsed and variant positions and the reads in which they occur are tallied

152 and grouped according to read UIDs. By default, common UID groups of 5 or more reads of

153 which at least $40 \%$ detect the same variant are classified as a variant 'super mutant' and variants

154 reported in two or more super mutants further classified as a 'super group'. This default value of

$15540 \%$ was chosen to allow successful super mutant detection even in the rare cases where a super

156 mutant was missed due to an identical UID tag being added to two distinct DNA molecules.

157 Examination of pilot data determined lowering this threshold did not add any false positive super

158 mutants and further, users of the software are able to override the defaults and determine the

159 appropriate value for each cut-off based on the nature of their dataset and the method by which it 
160 was generated. Finally, optional summary graphics of the variants in the context of sequence

161 read depth and chromosomal position are created using $\mathrm{R}$.

162

\section{Workflow implementation}

164 The overall workflow is comprised of discrete command-line interface calls to both custom- and open source-tools as well as UNIX utilities. All commands are stored within a configuration module and executed by a Perl wrapper script to allow chaining together of each command and

167 automation of the multifaceted workflow. This also allows for easy, frequent customisation of

168 workflow commands (should this be desired) and the capturing of specific commands and run-

169 level information into a log file that contributes to analysis reproducibility. The workflow has the

170 facility to allow it to be resumed or re-run from any point midway through the analysis.

171

172 The workflow commands, specifically, are various calls to several purpose-built tools

173 (implemented in Perl), external open-source bioinformatics software tools and UNIX utilities.

174 Custom Perl scripts are used to perform workflow steps to identify, remove and store UID tags

175 from each read, to aggregate and summarise variant calls within UID groups and to generate

176 final reports and graphs. Alignment of sequence reads is accomplished using BWA, variant

177 calling is done with SAMtools calmd, and graphing performed with R. Identification and

178 cleaning of reads containing runs of Ns is performed using sed and awk commands piped to

179 other UNIX utilities such as cut, sort, uniq, and cat which are required to manipulate the output

180 of these tools.

181

\section{Output}


183 The final report contains a listing of all variants detected, based on either the user-configured

184 expected variant frequency or default parameters (e.g. a common UID group must contain at

185 least five reads with at least $40 \%$ sharing the same variant). For each called variant, the output

186 super mutant summary reports the chromosome and genomic coordinate(s), the variant base, the

187 UID, the number of total variant reads in the groups, the number of reads in the group and the

188 fraction of variant reads. The super group report contains information on recurrent super mutants

189 (grouped by common genomic coordination and variant base) and additionally reports their

190 frequency.

191

192 Results and Discussion

193 We developed DeepSNVMiner to disambiguate tagged sequence groups within mixed cell

194 populations and detect sequence variants specific to individual amplified DNA molecules. To

195 assess the performance of DeepSNVMiner we first compare it to the well known variant callers

196 FreeBayes,( http://arxiv.org/abs/1207.3907), Genome analysis toolkit (GATK) (McKenna et al.

197 2010), SAMTools/BCFtools (Li 2011), and LoFreq (Wilm et al. 2012) using simulated tagged

198 sequence data at increasing variant dilution levels. Next, we test DeepSNVMiner by running a

199 dilution series with genomic DNA from two cells lines: one of which is known to contain a

200 known heterozygous somatic variant.

201

\section{Comparison with existing software}

203 To test the effectiveness of DeepSNVMiner at increasing dilution levels two datasets containing

204 100-bp paired-end reads from chromosome 22 of the human reference genome (GRCh37) were

205 created, with each read-pair having a randomly generated $10 \mathrm{bp}$ barcodes attached at the $5^{\prime}$ end to 
206 simulate the attachment of a UID sequence tag to the original DNA molecule. The first input

207 data set contained no mutations while the second input data set contained randomly generated

208 single nucleotide variants (SNVs) with each mutated read duplicated randomly between 1 to 50

209 times within the FASTQ files to simulate the polymerase chain reaction (PCR) replication

210 process of initial DNA fragments. Mixing the two data sets in appropriate concentrations

211 simulated dilution levels of $0 \%, 50 \%, 90 \%, 99 \%, 99.9 \%, 99.99 \%, 99.999 \%$, and $99.9999 \%$ with

$2124,000,000$ million total paired end reads ultimately added to each FASTQ file. For each dilution

213 level the FASTQ files were first aligned to chromosome 22 and variants called using

214 DeepSNVMiner, FreeBayes, GATK, SAMTools, and LoFreq run with default parameters or as

215 suggested in documentation (Table S1). False positive (Fig. 3) and false negative rates (Fig. 4)

216 were then calculated (Table S2)

217

218 Real data evaluation

219 To evaluate the performance of DeepSNVMiner on real data versus other variant callers, we 220 performed a dilution series using a mixture of genomic DNA from two cell lines HEK293 and

221 OCI-LY10 (Table S3). Cell line OCI-LY10 carries a heterozygous point mutation within the 222 MYD88 gene at L265P or chr3:38172641 (GRCh37), a somatic mutation occurring frequently in 223 non-Hodgkin lymphoma (Ngo et al. 2011). It would be clinically useful to have a method to 224 detect and enumerate rare cells carrying this mutation in samples of blood or bone marrow. For 225 each cell mixture in the dilution series, a 116bp genomic region surrounding chr3:38172641 was 226 amplified using primers with UID tags and sample ID tags and a per-sample average of 183 227 thousand paired-end reads were sequenced on an Illumina MiSeq. The resulting sequence reads 228 were analysed with DeepSNVMiner, FreeBayes, GATK, LoFreq, and SAMTools and the ability 
229 to detect the heterozygous mutation was measured.

230

231 DeepSNVMiner was successfully able to detect the mutation in dilution levels down to $1 / 1000$

232 compared to $1 / 100$ for LoFreq, $1 / 10$ for GATK, and only in the non-diluted sample for

233 FreeBayes and SAMTools (Table 1). In the non-diluted sample, DeepSNVMiner was able to

234 detect the mutation in 4055 separate super mutants consisting of 59038 total DNA sequences and 235 at the lower range of detection (1/1000), DeepSNVMiner detected the variant in 6 separate super

236 mutants consisting of 120 total DNA sequences (Fig. 5)

237 The mutation was reliably detected at concentrations of $1 / 1000$ by DeepSNVMiner but not in

238 concentrations of $1 / 10000$ indicating the lower detection limit lies somewhere in this range.

239 However, it should be noted this limit is imposed by current laboratory methodology however, as

240 DeepSNVMiner remains capable of achieving the theoretical limits of the technology imposed

241 by the chosen length of UID sequences.

242

243 Conclusions

244 We present DeepSNVMiner; an integrated tool set and automated workflow to allow robust and

245 reliable identification of sequence variants present in a subset of sequences within a tagged input

246 DNA sample. This tool makes available the analysis procedure required to support SafeSeqs and

247 similar UID tagged sequence datasets. DeepSNVMiner has been built to allow easy automation

248 and reproducibility and makes this technique available to a wide range of applications.

\section{Acknowledgements}


251 We thank the National Computational Infrastructure (Australia) for continued access to 252 significant computation resources and technical expertise.

253

\section{References}


255

256

257

258

259

260

261

262

263

264

265

266

267

268

269

270

271

272

273

274

275

276

277

278

279

280

281

282

283

284

285

286

287

288

289

290

291

292

293

294

295

296

297

298

299

300

Al-Mawsawi LQ, Wu NC, Olson C, Shi V, Qi H, Zheng X, Wu T, and Sun R. 2014. Highthroughput profiling of point mutations across the HIV-1 genome. Retrovirology 11:124.

Bidard FC, Weigelt B, and Reis-Filho JS. 2013. Going with the flow: from circulating tumor cells to DNA. Sci Transl Med 5:207ps214.

Dressman D, Yan H, Traverso G, Kinzler KW, and Vogelstein B. 2003. Transforming single DNA molecules into fluorescent magnetic particles for detection and enumeration of genetic variations. Proc Natl Acad Sci U S A 100:8817-8822.

Faust GG, and Hall IM. 2014. SAMBLASTER: fast duplicate marking and structural variant read extraction. Bioinformatics 30:2503-2505.

Field MA, Cho V, Andrews TD, and Goodnow CC. 2015. Reliably Detecting Clinically Important Variants Requires Both Combined Variant Calls and Optimized Filtering Strategies. PLoS One 10:e143199.

Forshew T, Murtaza M, Parkinson C, Gale D, Tsui DW, Kaper F, Dawson SJ, Piskorz AM, Jimenez-Linan M, Bentley D, Hadfield J, May AP, Caldas C, Brenton JD, and Rosenfeld N. 2012. Noninvasive identification and monitoring of cancer mutations by targeted deep sequencing of plasma DNA. Sci Transl Med 4:136ra168.

Fu GK, Hu J, Wang PH, and Fodor SP. 2011. Counting individual DNA molecules by the stochastic attachment of diverse labels. Proc Natl Acad Sci U S A 108:9026-9031.

Georgiou G, Ippolito GC, Beausang J, Busse CE, Wardemann H, and Quake SR. 2014. The promise and challenge of high-throughput sequencing of the antibody repertoire. Nat Biotechnol 32:158-168.

Hiatt JB, Pritchard CC, Salipante SJ, O'Roak BJ, and Shendure J. 2013. Single molecule molecular inversion probes for targeted, high-accuracy detection of low-frequency variation. Genome Res 23:843-854.

Jabara CB, Jones CD, Roach J, Anderson JA, and Swanstrom R. 2011. Accurate sampling and deep sequencing of the HIV-1 protease gene using a Primer ID. Proc Natl Acad Sci U S A 108:20166-20171.

Kinde I, Wu J, Papadopoulos N, Kinzler KW, and Vogelstein B. 2011. Detection and quantification of rare mutations with massively parallel sequencing. Proc Natl Acad Sci US A 108:9530-9535.

Kivioja T, Vaharautio A, Karlsson K, Bonke M, Enge M, Linnarsson S, and Taipale J. 2012. Counting absolute numbers of molecules using unique molecular identifiers. Nat Methods 9:72-74.

Li H. 2011. A statistical framework for SNP calling, mutation discovery, association mapping and population genetical parameter estimation from sequencing data. Bioinformatics 27:2987-2993.

Li H, and Durbin R. 2009. Fast and accurate short read alignment with Burrows-Wheeler transform. Bioinformatics 25:1754-1760.

Li H, Handsaker B, Wysoker A, Fennell T, Ruan J, Homer N, Marth G, Abecasis G, Durbin R, and Genome Project Data Processing S. 2009. The Sequence Alignment/Map format and SAMtools. Bioinformatics 25:2078-2079.

McKenna A, Hanna M, Banks E, Sivachenko A, Cibulskis K, Kernytsky A, Garimella K, Altshuler D, Gabriel S, Daly M, and DePristo MA. 2010. The Genome Analysis Toolkit: a MapReduce framework for analyzing next-generation DNA sequencing data. Genome Res 20:1297-1303.

Ngo VN, Young RM, Schmitz R, Jhavar S, Xiao W, Lim KH, Kohlhammer H, Xu W, Yang Y, 
301

302

303

304

305

306

307

308

309

310

311

312

313

314

315

316

317

318

319

320

321
Zhao H, Shaffer AL, Romesser P, Wright G, Powell J, Rosenwald A, Muller-Hermelink HK, Ott G, Gascoyne RD, Connors JM, Rimsza LM, Campo E, Jaffe ES, Delabie J, Smeland EB, Fisher RI, Braziel RM, Tubbs RR, Cook JR, Weisenburger DD, Chan WC, and Staudt LM. 2011. Oncogenically active MYD88 mutations in human lymphoma. Nature 470:115-119.

Ottesen EA, Hong JW, Quake SR, and Leadbetter JR. 2006. Microfluidic digital PCR enables multigene analysis of individual environmental bacteria. Science 314:1464-1467.

Ross MG, Russ C, Costello M, Hollinger A, Lennon NJ, Hegarty R, Nusbaum C, and Jaffe DB. 2013. Characterizing and measuring bias in sequence data. Genome Biol 14:R51.

Schirmer M, Ijaz UZ, D'Amore R, Hall N, Sloan WT, and Quince C. 2015. Insight into biases and sequencing errors for amplicon sequencing with the Illumina MiSeq platform. Nucleic Acids Res 43:e37.

Schmitt MW, Kennedy SR, Salk JJ, Fox EJ, Hiatt JB, and Loeb LA. 2012. Detection of ultra-rare mutations by next-generation sequencing. Proc Natl Acad Sci U S A 109:14508-14513.

Vogelstein B, and Kinzler KW. 1999. Digital PCR. Proc Natl Acad Sci U S A 96:9236-9241.

Wilm A, Aw PP, Bertrand D, Yeo GH, Ong SH, Wong CH, Khor CC, Petric R, Hibberd ML, and Nagarajan N. 2012. LoFreq: a sequence-quality aware, ultra-sensitive variant caller for uncovering cell-population heterogeneity from high-throughput sequencing datasets. Nucleic Acids Res 40:11189-11201. 
1

DeepSNVMiner Barcode and Adaptor Processing

A sample sequence read which has undergone molecule tagging with a ten base pair UID consisting of five bases each attached to the 5' and 3' end. DeepSNVMiner first removes any adapters followed by the removal of the unique sequence identifier (UID) from the raw sequence data. The UID sequence information is preserved in the FASTQ header allowing for variant detection on read groups sharing a common UID.

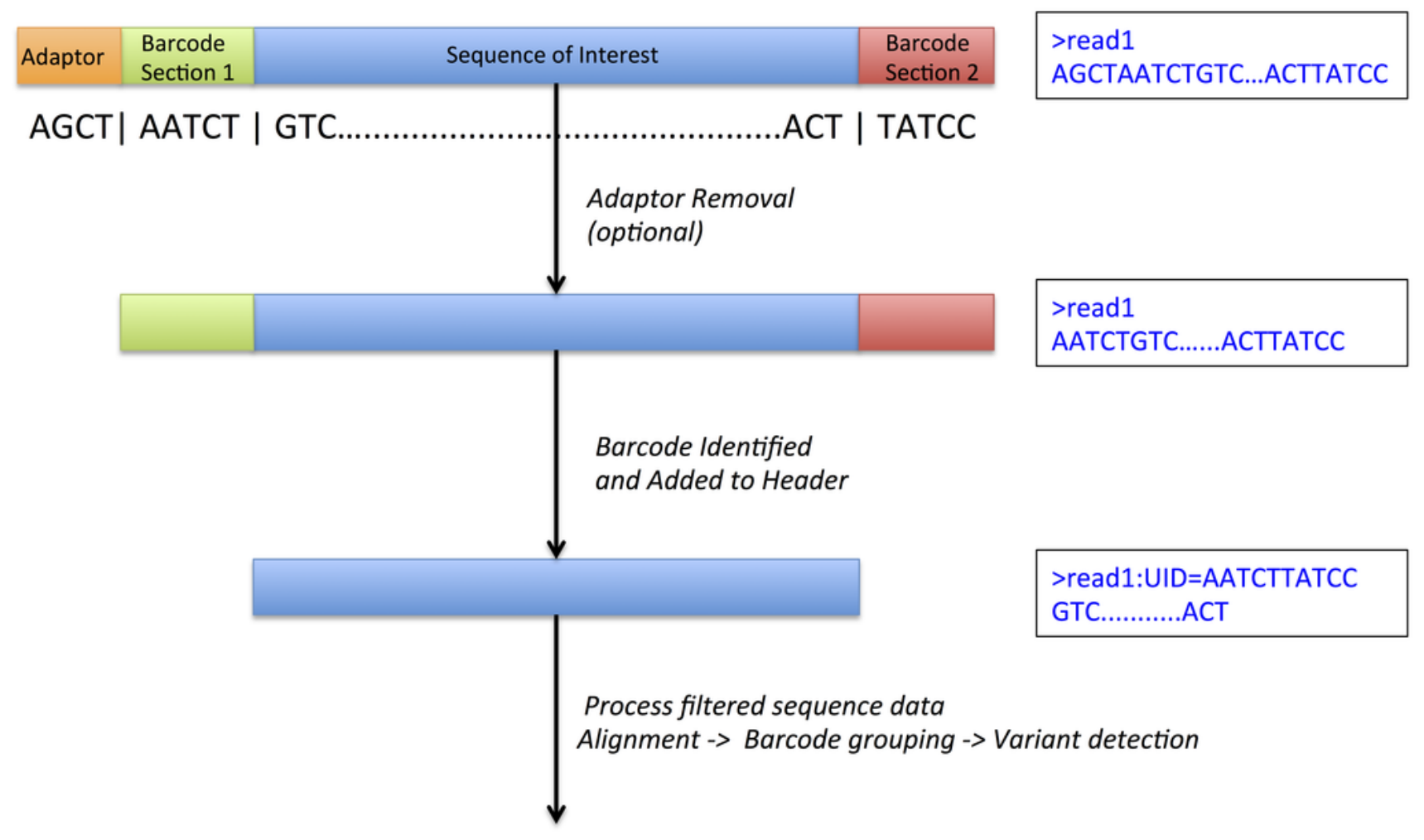


2

DeepSNVMiner Workflow

DeepSNVMiner Workflow consists of seven major steps: UID definition, sequence data QC, UID processing and grouping, alignment, variant detection, report generation, and optionally graphing. 


\section{New barcode sequence data}

-Define barcode / coordinates

QC sequence data

-Filter out all $N$ reads

Group reads by barcode

-Append barcode to read header

Align reads

-Sorted BAM with BWA/samtools

\section{Call variants}

-Detect SNVs / group by barcode

Generate reports

-Generate super mutant report

Generate graphs (optional)

-Supermutant location graphs 
3

Variant Caller False Positive Rate at Increased Dilution Levels

\section{Variant Caller False Positive Rates at Increased Dilutions}

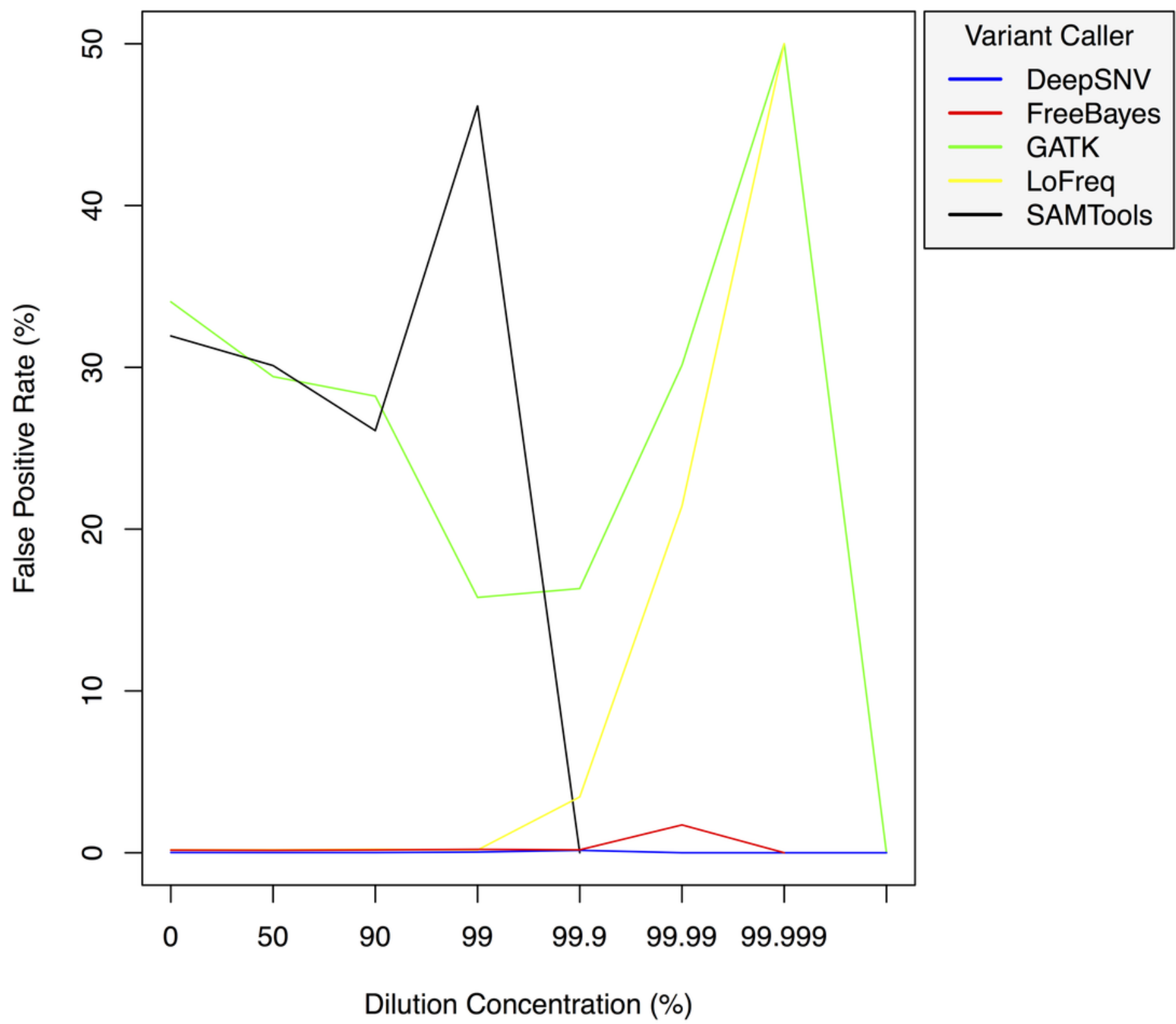


4

Variant Caller False Negative Rate at Increased Dilution Levels

False negative rates for DeepSNVMiner compared to FreeBayes, GATK, LoFreq, and SAMTools at increasing variant dilution levels using synthetic data.

\section{Variant Caller False Negative Rates at Increased Dilutions}

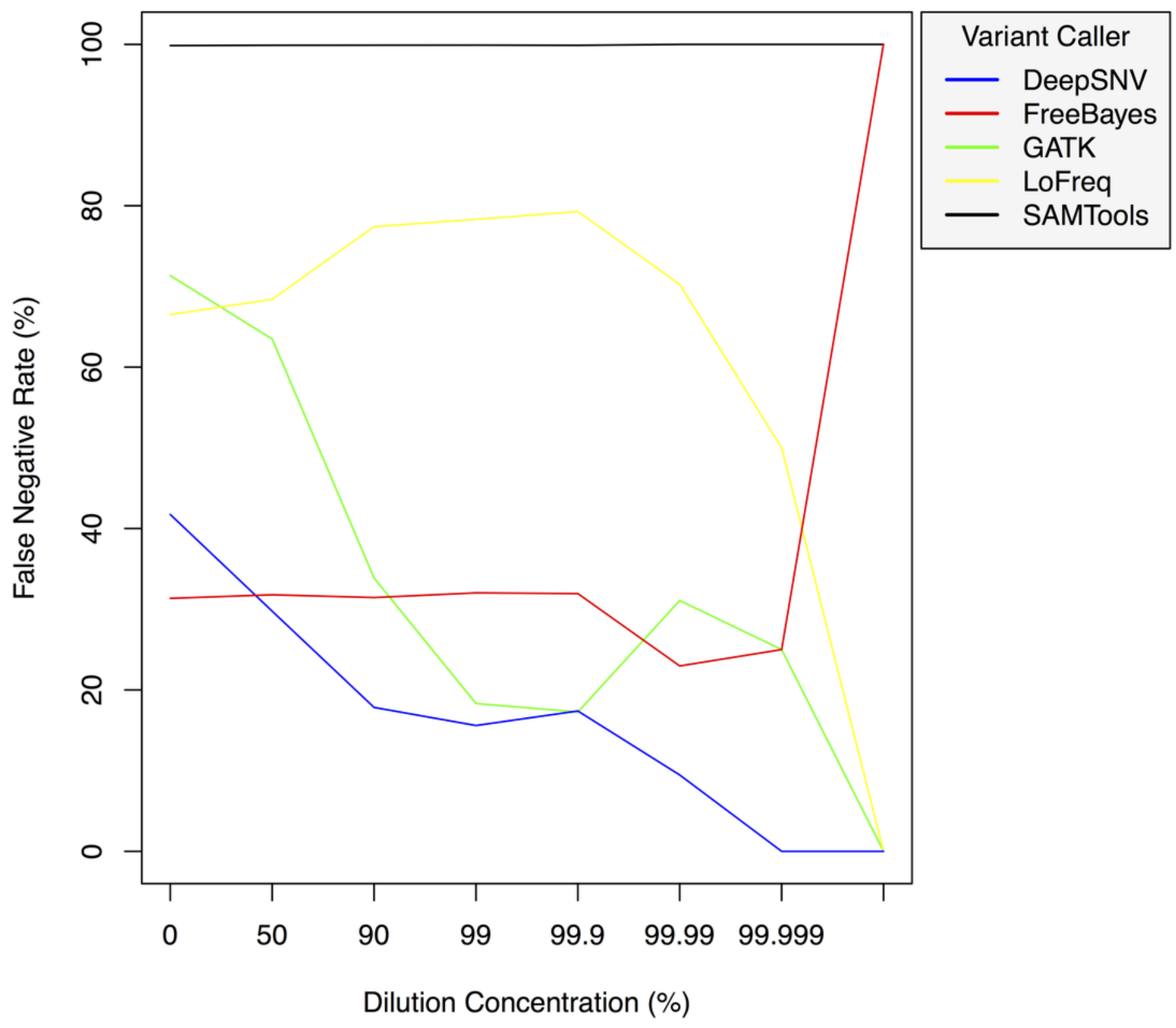




\section{Table $\mathbf{1}$ (on next page)}

Variant Caller Ability to Detect Known Heterozygous Mutation at Different Dilution Levels

A dilution series was performed with genomic DNA from two cells lines: HEK293 containing wild-type MYD88 and OCI-LY10 containing known heterozygous L265P MYD88 mutation. The ability to detect the known heterozygous mutation was determined for DeepSNVMiner, FreeBayes, GATK, LoFreq, and SAMTools at increasing dilution levels. 


\begin{tabular}{|l|l|l|l|l|l|}
\hline $\begin{array}{l}\text { Dilution } \\
\text { Percent }\end{array}$ & $\begin{array}{l}\text { Deep- } \\
\text { SNVMiner }\end{array}$ & FreeBayes & GATK & LoFreq & SAMTools \\
\hline 0 & Detected & Detected & Detected & Detected & Detected \\
\hline 90 & Detected & Not detected & Detected & Detected & Not detected \\
\hline 99 & Detected & Not detected & Not detected & Detected & Not detected \\
\hline 99.9 & Detected & Not detected & Not detected & Not detected & Not detected \\
\hline 99.99 & Not detected & Not detected & Not detected & Not detected & Not detected \\
\hline 99.999 & Not detected & Not detected & Not detected & Not detected & Not detected \\
\hline 99.9999 & Not detected & Not detected & Not detected & Not detected & Not detected \\
\hline
\end{tabular}




\section{5}

\section{DeepSNVMiner Dilution Series Results}

To measure the sensitivity of DeepSNVMiner a dilution series containing known heterozygous L265P MYD88 mutation was performed. DeepSNVMiner was able to detect the mutation in a sample where only 1 in 1000 samples contained the mutation.

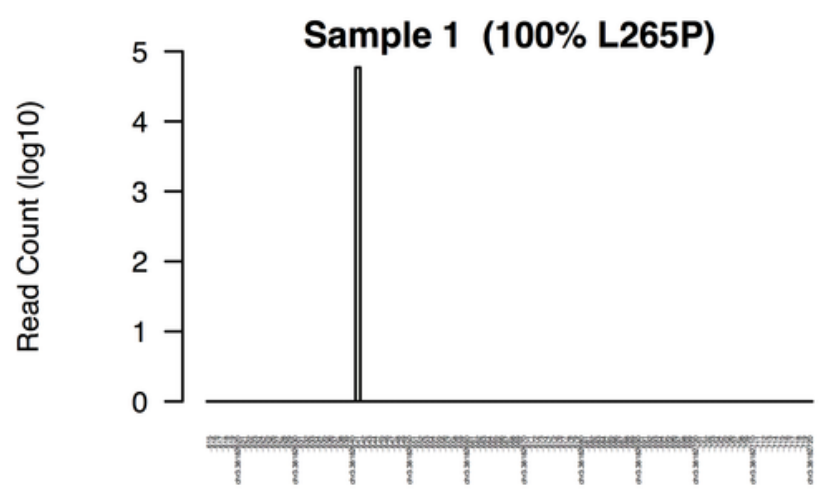

Genomic Coordinates

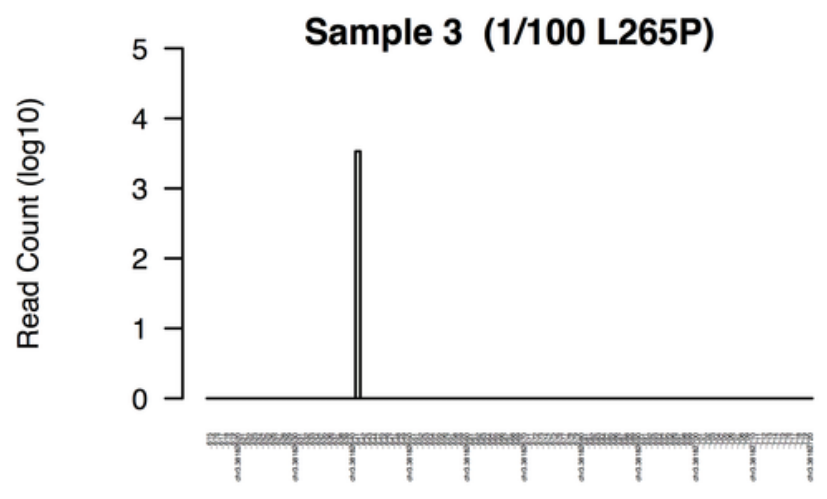

Genomic Coordinates

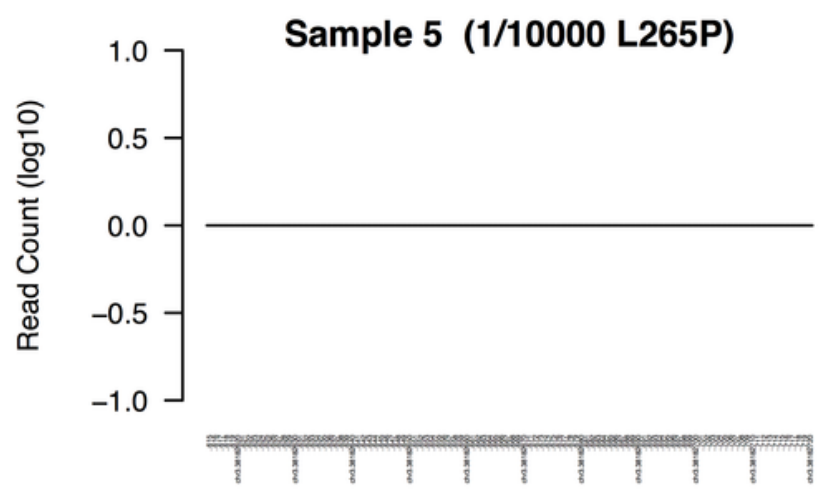

Genomic Coordinates

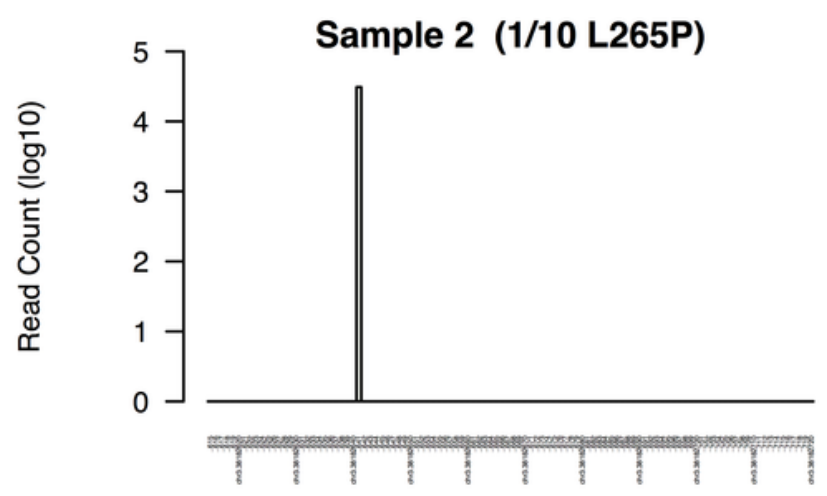

Genomic Coordinates

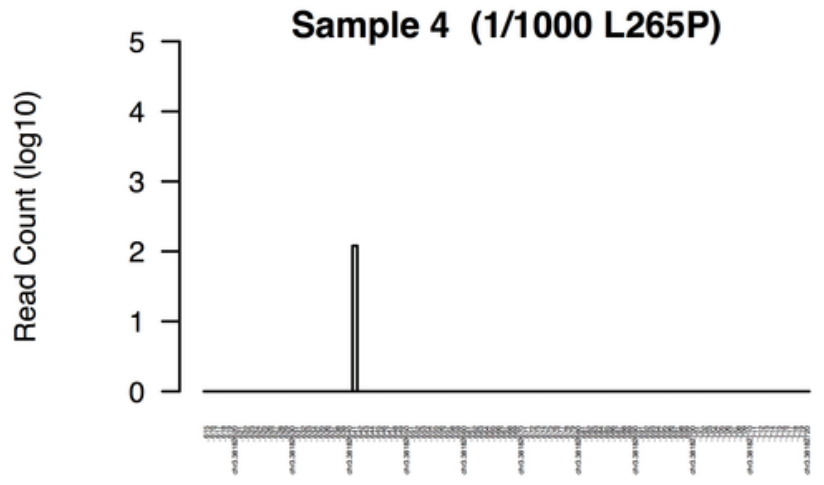

Genomic Coordinates

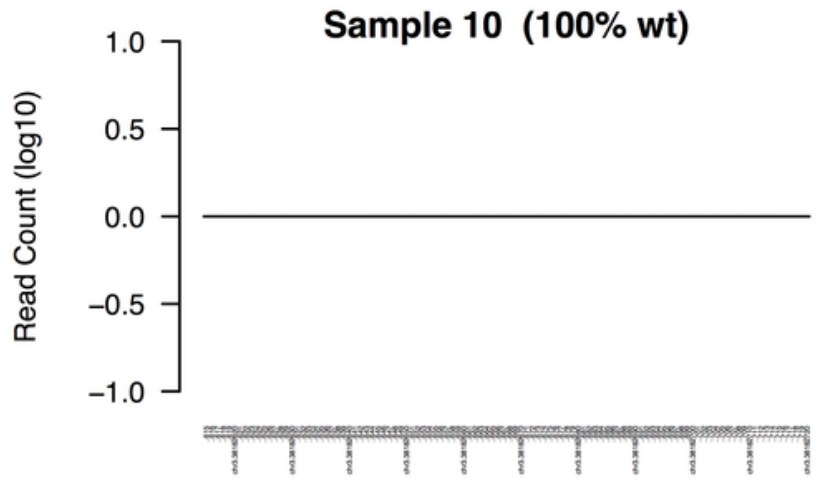

Genomic Coordinates 